\title{
INVERSE MEDIUM SCATTERING PROBLEMS FOR ELECTROMAGNETIC WAVES*
}

\author{
GANG $\mathrm{BAO}^{\dagger}$ AND PEIJUN $\mathrm{LI}^{\dagger}$
}

\begin{abstract}
Consider a time-harmonic electromagnetic plane wave incident on a medium enclosed by a bounded domain in $\mathbb{R}^{3}$. In this paper, existence and uniqueness of the variational problem for forward scattering are established. An energy estimate for the scattered field with a uniform bound with respect to the wavenumber is obtained in the case of low frequency on which the Born approximation is based. A continuation method for the inverse medium scattering problem, which reconstructs the scatterer of an inhomogeneous medium from boundary measurements of the scattered wave, is developed. The algorithm requires multifrequency scattering data. Using an initial guess from the Born approximation, each update is obtained via recursive linearization on the wavenumber $k$ by solving one forward problem and one adjoint problem of Maxwell's equations.
\end{abstract}

Key words. inverse medium scattering, Maxwell's equations, recursive linearization

AMS subject classifications. $65 \mathrm{~N} 21,78 \mathrm{~A} 46$

DOI. $10.1137 / 040607435$

1. Introduction. Consider the systems of time-harmonic Maxwell's equations in three dimensions

$$
\begin{aligned}
\nabla \times E^{t} & =\mathrm{i} \omega \mu^{*} H^{t}, \\
\nabla \times H^{t} & =-\mathrm{i} \omega \varepsilon^{*} E^{t},
\end{aligned}
$$

where $E^{t}$ and $H^{t}$ are the total electric field and magnetic field, respectively; $\omega>0$ is the frequency; and $\varepsilon^{*}$ and $\mu^{*}$ are the electric permittivity and the magnetic permeability, respectively. Denote by $\varepsilon_{0}>0, \mu_{0}>0$ the permittivity and permeability of the vacuum. The fields are further assumed to be nonmagnetic; i.e., $\mu^{*}=\mu_{0}$. Rewriting $\varepsilon^{*}=\varepsilon_{0} \varepsilon, \varepsilon=1+q(x)$ is the relative permittivity, where $q(x)$ is the scatterer, which is assumed to have a compact support, and $\mathfrak{R}(q(x))>-1$.

Taking the curl of (1.1) and eliminating the magnetic field $H^{t}$, we obtain the uncoupled equation for the electric field $E^{t}$ :

$$
\nabla \times\left(\nabla \times E^{t}\right)-k^{2} \varepsilon E^{t}=0
$$

where $k=\omega \sqrt{\varepsilon_{0} \mu_{0}}$ is called the wavenumber, satisfying $0<k_{\min } \leq k \leq k_{\max }<\infty$. The total electric field $E^{t}$ consists of the incident field $E^{i}$ and the scattered field $E$ :

$$
E^{t}=E^{i}+E .
$$

Assume that the incident field is a plane wave of the normalized form [5]

$$
E^{i}=\mathrm{i} k \vec{p} e^{\mathrm{i} k x \cdot \vec{n}}
$$

*Received by the editors April 27, 2004; accepted for publication (in revised form) March 11, 2005; published electronically August 9, 2005. This research was supported in part by NSF grant DMS 01-04001 and ONR grant N000140210365. Preliminary progress of this research was announced in [3].

http://www.siam.org/journals/siap/65-6/60743.html

${ }^{\dagger}$ Department of Mathematics, Michigan State University, East Lansing, MI 48824-1027 (bao@ math.msu.edu, lipeijun@math.msu.edu). 
where $\vec{n} \in \mathbb{S}^{2}$ is the propagation direction and $\vec{p} \in \mathbb{S}^{2}$ is the polarization satisfying $\vec{p} \cdot \vec{n}=0$. Evidently, such an incident wave satisfies the homogeneous equation

$$
\nabla \times\left(\nabla \times E^{i}\right)-k^{2} E^{i}=0 .
$$

It follows from (1.3) and (1.5) that the scattered field satisfies

$$
\nabla \times(\nabla \times E)-k^{2} \varepsilon E=k^{2} q(x) E^{i} .
$$

In addition, the scattered field is required to satisfy the following Silver-Müller radiation condition:

$$
\lim _{r \rightarrow \infty} r\left[\nabla \times E \times \frac{x}{r}-\mathrm{i} k E\right]=0,
$$

where $r=|x|$. In practice, it is convenient to reduce the problem to a bounded domain by introducing an artificial surface. Let $\Omega$ be the compact support of the scatterer $q(x)$. Assume that $R>0$ is a constant such that the support of the scatterer, $\Omega$, is included in the ball $B=\left\{x \in \mathbb{R}^{3}:|x|<R\right\}$. Let $S$ be the sphere of the ball, i.e., $S=\left\{x \in \mathbb{R}^{3}:|x|=R\right\}$. Denote by $\nu$ the outward unit normal to $S$. A suitable boundary condition then has to be imposed on $S$. For simplicity, we employ the first order absorbing boundary condition (impedance boundary condition) [12] as

$$
\nu \times(\nabla \times E)+\mathrm{i} k \nu \times(\nu \times E)=0 \quad \text { on } S .
$$

Given the incident field $E^{i}$, the forward problem is to determine the scattered field $E$ for the known scatterer $q(x)$, which is assumed further to be in $L^{\infty}(B)$. Based on the Helmholtz decomposition and a compact imbedding result, the forward problem is shown to have a unique solution for all but possibly a discrete set of wavenumbers. Furthermore, an energy estimate for the scattered field, with a uniform bound with respect to the wavenumber, is given in the low frequency case. The estimate provides a theoretical basis for our linearization algorithm. For numerical solution of the forward scattering problem in an open domain, the reader is referred to $[14,15,16,21]$ and references therein. The inverse medium scattering problem is to determine the scatterer $q(x)$ from the measurements of near field current densities, the tangential trace of the scattered field $\nu \times\left. E\right|_{S}$, given the incident field. Although this is a classical problem in inverse scattering theory, little is known on reconstruction methods, especially in the three dimensional case, due to the nonlinearity, ill-posedness, and large scale computation associated with the inverse scattering problem. We refer the reader to $[1,6,10,11,23]$ for related results on the inverse medium problem. See [5] for an account of recent progress on the general inverse scattering problem.

The goal of this work is to present a recursive linearization method that solves the inverse medium scattering problem of Maxwell's equations in three dimensions. The reader is referred to $[2,4]$ for recursive linearization approaches for solving the inverse medium scattering problems in two dimensions. Our algorithm requires multifrequency scattering data, and the recursive linearization is obtained by a continuation method on the wavenumber. It first solves a linear equation (Born approximation) at the lowest wavenumber, which may be done by using the fast Fourier transform (FFT). Updates are subsequently obtained by using higher and higher wavenumbers. Following the idea of the Kaczmarz method [6, 18, 19], we use partial data to perform the nonlinear Landweber iteration at each wavenumber. For each iteration, one forward and one adjoint state of Maxwell's equations are solved, which may be implemented by using the symmetric second order edge (Nédélec) elements. 
The plan of this paper is as follows. Analysis of the variational problem for forward scattering is presented in section 2. Based on the Helmholtz decomposition, a compact imbedding result, and the Lax-Milgram lemma, the well-posedness of the forward scattering is proved. An important energy estimate is given. Section 3 is devoted to the numerical study of inverse medium scattering. Using the initial guess of the reconstruction derived from the Born approximation, a regularized iterative linearization algorithm is proposed. Numerical examples are presented in section 4. The paper is concluded with some remarks and future directions in section 5 .

2. Analysis of the variational problem for forward scattering. In this section, the variational formulation for the forward scattering problem is discussed. The analysis provides a criterion for weak scattering, which plays an important role in the inversion algorithm.

To state our boundary value problem, following [17], we first introduce the standard Sobolev spaces:

$$
\begin{aligned}
& L_{t}^{2}(S)=\left\{u \in\left(L^{2}(S)\right)^{3}: \nu \cdot u=0 \text { on } S\right\} \\
& H_{0}^{1}(B)=\left\{u \in H^{1}(B): u=0 \text { on } S\right\} \\
& H(\operatorname{curl}, B)=\left\{u \in\left(L^{2}(B)\right)^{3}: \nabla \times u \in\left(L^{2}(B)\right)^{3}\right\}, \\
& H_{\mathrm{imp}}(\operatorname{curl}, B)=\left\{u \in H(\operatorname{curl}, B): \nu \times u \in L_{t}^{2}(S)\right\},
\end{aligned}
$$

where $H_{\mathrm{imp}}(\operatorname{curl}, B)$ is an appropriate subspace of $H(\operatorname{curl}, B)$ for solving problems involving the impedance boundary condition. Correspondingly, these spaces are equipped with the norms

$$
\begin{aligned}
& \|u\|_{L_{t}^{2}(S)}=\|u\|_{\left(L^{2}(S)\right)^{3}}, \\
& \|u\|_{H^{1}(B)}^{2}=\|u\|_{L^{2}(B)}^{2}+\|\nabla u\|_{\left(L^{2}(B)\right)^{3}}^{2}, \\
& \|u\|_{H(\operatorname{curl}, B)}^{2}=\|u\|_{\left(L^{2}(B)\right)^{3}}^{2}+\|\nabla \times u\|_{\left(L^{2}(B)\right)^{3}}^{2}, \\
& \|u\|_{H_{\text {imp }}(\operatorname{curl}, B)}^{2}=\|u\|_{H(\operatorname{curl}, B)}^{2}+\|\nu \times u\|_{L_{t}^{2}(S)}^{2} .
\end{aligned}
$$

For convenience, denote the $\left(L^{2}(B)\right)^{3}$ and $\left(L^{2}(S)\right)^{3}$ inner products by

$$
(u, v)=\int_{B} u \cdot \bar{v} d x \quad \text { and } \quad\langle u, v\rangle=\int_{S} u \cdot \bar{v} d s,
$$

respectively, where the overline denotes the complex conjugate. Introduce the bilinear form $a: H_{\mathrm{imp}}(\operatorname{curl}, B) \times H_{\mathrm{imp}}(\operatorname{curl}, B) \rightarrow \mathbb{C}$,

$$
a(E, \phi)=(\nabla \times E, \nabla \times \phi)-k^{2}(\varepsilon E, \phi)+\mathrm{i} k\langle\nu \times E, \nu \times \phi\rangle,
$$

and the linear functional on $H_{\mathrm{imp}}(\operatorname{curl}, B)$,

$$
b(\phi)=k^{2}\left(q E^{i}, \phi\right) .
$$

Then we have the weak form of the boundary value problem (1.6) and (1.7): find $E \in H_{\text {imp }}(\operatorname{curl}, B)$ such that

$$
a(E, \phi)=b(\phi) \quad \forall \phi \in H_{\mathrm{imp}}(\operatorname{curl}, B) .
$$

Throughout the paper, $C$ stands for a positive generic constant, whose value may change step by step but should always be clear from the context. 
Before presenting the main result for the variational problem, we state several useful lemmas. The reader is referred to [17] for detailed discussions and proofs.

Lemma 2.1 (Helmholtz decomposition). The spaces $X$ and $Y$ are closed subspaces of $H_{\mathrm{imp}}(\mathrm{curl}, B)$, which is the direct sum of the spaces $X$ and $Y$; i.e.,

$$
H_{\text {imp }}(\operatorname{curl}, B)=X \oplus Y \text {. }
$$

Here

$$
X=\left\{u \in H_{\mathrm{imp}}(\operatorname{curl}, B): \operatorname{div}(\varepsilon u)=0 \quad \text { in } B\right\}
$$

and

$$
Y=\left\{\nabla \xi: \xi \in H_{0}^{1}(B)\right\} .
$$

Lemma 2.2 (compact imbedding). The space $X$ is compactly imbedded into the space $\left(L^{2}(B)\right)^{3}$.

Lemma 2.3 (Friedrichs inequality). There exists a positive constant $C$, independent of the wavenumber, such that for all $u \in X$

$$
\|u\|_{\left(L^{2}(B)\right)^{3}} \leq C\left(\|\nabla \times u\|_{\left(L^{2}(B)\right)^{3}}+\|\nu \times u\|_{\left(L^{2}(S)\right)^{3}}\right) .
$$

Next we prove the well-posedness of the variational problem (2.1) and obtain an energy estimate for the scattered field with a uniform bound with respect to the wavenumber in the case of low frequency.

THEOREM 2.1. If the wavenumber is sufficiently small, the variational problem (2.1) admits a unique weak solution in $H_{\mathrm{imp}(\operatorname{curl}, B)}$ given by $E=u+\nabla p$, while $u \in X, p \in H_{0}^{1}(B)$. Furthermore, we have the estimate

$$
\|E\|_{H_{\mathrm{imp}(\mathrm{curl}, B)}} \leq C k|\Omega|^{1 / 2}\|q\|_{L^{\infty}(B)},
$$

where the constant $C$ is independent of $k$ and $\Omega$ is the compact support of the scatterer.

Proof. Using the Helmholtz decomposition, we take $E=u+\nabla p$ and $\phi=v+\nabla \xi$, for any $v \in X, \xi \in H_{0}^{1}(B)$. Observe that $a(u, \nabla \xi)=0$, for any $\xi \in H_{0}^{1}(B)$, by the definition of $X$. Therefore, we decompose the variational equation (2.1) into the form

$$
a(u, v)+a(\nabla p, v)+a(\nabla p, \nabla \xi)=b(v)+b(\nabla \xi) \quad \forall v \in X, \xi \in H_{0}^{1}(B) .
$$

First, we determine $p \in H_{0}^{1}(B)$ by the solution of

$$
a(\nabla p, \nabla \xi)=b(\nabla \xi) \quad \forall \xi \in H_{0}^{1}(B),
$$

which gives explicitly

$$
-(\varepsilon \nabla p, \nabla \xi)=\left(q E^{i}, \nabla \xi\right) \quad \forall \xi \in H_{0}^{1}(B) .
$$

The existence and uniqueness of the solution $p$ in $H_{0}^{1}(B)$ may be proved by a direct application of the Lax-Milgram lemma with the estimate

$$
\|\nabla p\|_{\left(L^{2}(B)\right)^{3}} \leq C k|\Omega|^{1 / 2}\|q\|_{L^{\infty}(B) .}
$$

Rewrite (2.3) as

$$
a(u, v)=b(v)-a(\nabla p, v) \quad \forall v \in X
$$


and decompose the bilinear form $a$ into $a=a_{1}+k^{2} a_{2}$, where

$$
\begin{aligned}
& a_{1}(u, v)=(\nabla \times u, \nabla \times v)+\mathrm{i} k\langle\nu \times u, \nu \times v\rangle, \\
& a_{2}(u, v)=-(\varepsilon u, v) .
\end{aligned}
$$

Using the inequality of arithmetic and geometric means, we conclude from Lemma 2.3 that $a_{1}$ is coercive:

$$
\begin{gathered}
\left|a_{1}(u, u)\right| \geq C k\left(\|\nabla \times u\|_{\left(L^{2}(B)\right)^{3}}^{2}+\|\nu \times u\|_{\left(L^{2}(S)\right)^{3}}^{2}\right) \geq C k\|u\|_{H_{\text {imp }}(\operatorname{curl}, B)}^{2} \\
\forall u \in X .
\end{gathered}
$$

The continuity of the bilinear form $a_{1}$ follows from the Cauchy-Schwarz inequality.

Next we prove the compactness of $a_{2}$. Define an operator $\mathcal{A}:\left(L^{2}(B)\right)^{3} \rightarrow X$ by

$$
a_{1}(\mathcal{A} u, v)=a_{2}(u, v) \quad \forall v \in X,
$$

which gives

$$
(\nabla \times \mathcal{A} u, \nabla \times v)+\mathrm{i} k\langle\nu \times \mathcal{A} u, \nu \times v\rangle=-(\varepsilon u, v) \quad \forall v \in X .
$$

Using the Lax-Milgram lemma again, it follows that

$$
\|\mathcal{A} u\|_{H_{\mathrm{imp}}(\operatorname{curl}, B)} \leq \frac{C}{k}\|u\|_{\left(L^{2}(B)\right)^{3}}
$$

where the constant $C$ is independent of $k$. Thus $\mathcal{A}$ is bounded from $\left(L^{2}(B)\right)^{3}$ to $X$, and $X$ is compactly imbedded into $\left(L^{2}(B)\right)^{3}$. Hence $\mathcal{A}:\left(L^{2}(B)\right)^{3} \rightarrow\left(L^{2}(B)\right)^{3}$ is a compact operator.

Define a function $w \in\left(L^{2}(B)\right)^{3}$ by requiring $w \in X$ and satisfying

$$
a_{1}(w, v)=b(v)-a(\nabla p, v) \quad \forall v \in X .
$$

More specifically, we have by using the Stokes formula that

$$
a_{1}(w, v)=k^{2}\left(q E^{i}, v\right)+k^{2}(\varepsilon \nabla p, v) \quad \forall v \in X .
$$

It follows from the Lax-Milgram lemma that

$$
\|w\|_{H_{\mathrm{imp}}(\operatorname{curl}, B)} \leq C\left(k^{2}|\Omega|^{1 / 2}\|q\|_{L^{\infty}(B)}+k\|\nabla p\|_{\left.\left(L^{2}(B)\right)^{3}\right)} .\right.
$$

An application of (2.4) yields

$$
\|w\|_{H_{\mathrm{imp}}(\operatorname{curl}, B)} \leq C k^{2}|\Omega|^{1 / 2}\|q\|_{L^{\infty}(B)} .
$$

Using the operator $\mathcal{A}$, we can see that the problem (2.5) is equivalent to finding $u \in\left(L^{2}(B)\right)^{3}$ such that

$$
\left(\mathcal{I}+k^{2} \mathcal{A}\right) u=w .
$$

When the wavenumber $k$ is small enough, the operator $\mathcal{I}+k^{2} \mathcal{A}$ has a uniformly bounded inverse. We then have the estimate

$$
\|u\|_{\left(L^{2}(B)\right)^{3}} \leq C\|w\|_{\left(L^{2}(B)\right)^{3}},
$$


where the constant $C$ is independent of $k$. However, rearranging (2.8), we have $u=$ $w-k^{2} \mathcal{A} u$, so $u \in X$ and, by the estimate (2.6) for the operator $\mathcal{A}$, we have

$$
\|u\|_{H_{\mathrm{imp}}(\operatorname{curl}, B)} \leq\|w\|_{H_{\mathrm{imp}}(\operatorname{curl}, B)}+C k\|u\|_{\left(L^{2}(B)\right)^{3}} .
$$

Combining the estimates (2.9) and (2.7) leads to

$$
\|u\|_{H_{\mathrm{imp}}(\operatorname{curl}, B)} \leq C k^{2}|\Omega|^{1 / 2}\|q\|_{L^{\infty}(B)} .
$$

Finally, it follows from the definition of the norm in $H_{\text {imp }}(\operatorname{curl}, B)$ that

$$
\|E\|_{H_{\mathrm{imp}}(\operatorname{curl}, B)} \leq\|u\|_{H_{\mathrm{imp}}(\operatorname{curl}, B)}+\|\nabla p\|_{\left(L^{2}(B)\right)^{3}} .
$$

The proof is complete by noting the estimates (2.10) and (2.4) for sufficiently small wavenumbers.

Remark 2.1. The energy estimate of the scattered field (2.2) provides a criterion for weak scattering. From this estimate, it is easily seen that, fixing any two of the three quantities, i.e., the wavenumber, the compact support of the scatterer $\Omega$, and the $L^{\infty}(B)$ norm of the scatterer, the scattering is weak when the third one is small. Especially for the given scatterer $q(x)$, i.e., the norm and the compact support are fixed, the scattering is weak when the wavenumber is small.

Remark 2.2. For a general wavenumber, from (2.8) the uniqueness and existence follow from the Fredholm alternative. If the scatterer $q(x)$ is more regular, say of $C_{0}^{2}(B)$ [8], unique continuation may be used to prove the uniqueness and thus the existence of the forward scattering problem (1.6), (1.7) for all $k>0$. Otherwise, if $k^{2}$ is not the eigenvalue for Maxwell's equations in the domain $B$, then the operator $\mathcal{I}+k^{2} \mathcal{A}$ has a bounded inverse. However, the bound depends on the wavenumber. Therefore, the constant $C$ in the estimate (2.2) depends on the wavenumber.

From the above discussion, we have the following theorem on the well-posedness of the variational problem (2.1).

THEOREM 2.2. Given the scatterer $q \in L^{\infty}(B)$, for all but possibly a discrete set of wavenumbers, the variational problem (2.1) admits a unique weak solution in $H_{\mathrm{imp}}(\operatorname{curl}, B)$, given by $E=u+\nabla p$, while $u \in X, p \in H_{0}^{1}(B)$.

3. Inverse medium scattering. In this section, a regularized recursive linearization method for solving the inverse medium scattering problem of Maxwell's equations in three dimensions is proposed. The algorithm, obtained by a continuation method on the wavenumber, requires multifrequency scattering data. At each wavenumber, the algorithm determines a forward model which produces the prescribed scattering data. At a low wavenumber, the scattered field is weak. Consequently, the nonlinear equation becomes essentially linear, known as the Born approximation. The algorithm first solves this nearly linear equation at the lowest wavenumber to obtain low-frequency modes of the true scatterer. The approximation is then used to linearize the nonlinear equation at the next higher wavenumber to produce a better approximation which contains more modes of the true scatterer. This process is continued until a sufficiently high wavenumber, where the dominant modes of the scatterer are essentially recovered.

3.1. Low-frequency modes of the scatterer. Rewrite (1.6) as

$$
\nabla \times(\nabla \times E)-k^{2} E=k^{2} q(x)\left(E^{i}+E\right),
$$


where the incident wave is taken as $E^{i}=\mathrm{i} k \vec{p}_{1} e^{\mathrm{i} k x \cdot \vec{n}_{1}}$. Consider a test function $F=$ $\mathrm{i} k \vec{p}_{2} e^{\mathrm{i} k x \cdot \vec{n}_{2}}$, where $\vec{p}_{2}, \vec{n}_{2} \in \mathbb{S}^{2}$ satisfy $\vec{p}_{2} \cdot \vec{n}_{2}=0$. Hence $F$ satisfies (1.5).

Multiplying (3.1) by $F$ and integrating over $B$ on both sides, we have

$$
\int_{B} F \cdot[\nabla \times(\nabla \times E)] d x-k^{2} \int_{B} F \cdot E d x=k^{2} \int_{B} q(x) F \cdot E^{i} d x+k^{2} \int_{B} q(x) F \cdot E d x .
$$

Integration by parts yields

$$
\begin{array}{r}
\int_{B} E \cdot[\nabla \times(\nabla \times F)] d x+\int_{S}[E \times(\nabla \times F)-F \times(\nabla \times E)] \cdot \nu d s-k^{2} \int_{B} F \cdot E d x \\
=k^{2} \int_{B} q(x) F \cdot E^{i} d x+k^{2} \int_{B} q(x) F \cdot E d x
\end{array}
$$

We have, by noting (1.5),

$$
\int_{S}[E \times(\nabla \times F)-F \times(\nabla \times E)] \cdot \nu d s=k^{2} \int_{B} q(x) F \cdot E^{i} d x+k^{2} \int_{B} q(x) F \cdot E d x .
$$

Using the boundary condition (1.7) of the scattered field and the special form of the incident wave $E^{i}$ and $F$, we get

$$
\begin{array}{r}
-\int_{S}(\nu \times E) \cdot\left(\vec{n}_{2} \times \vec{p}_{2}\right) e^{\mathrm{i} k x \cdot \vec{n}_{2}} d s+\int_{S}[\nu \times(\nu \times E)] \cdot \vec{p}_{2} e^{\mathrm{i} k x \cdot \vec{n}_{2}} d s \\
=\int_{B} q(x) F \cdot E^{i} d x+\int_{B} q(x) F \cdot E d x .
\end{array}
$$

A simple calculation yields

$$
\begin{aligned}
\int_{B} q(x) e^{\mathrm{i} k x \cdot\left(\vec{n}_{1}+\vec{n}_{2}\right)} d x= & \frac{1}{\left(\vec{p}_{1} \cdot \vec{p}_{2}\right) k^{2}} \int_{S}(\nu \times E) \cdot\left(\vec{n}_{2} \times \vec{p}_{2}+\nu \times \vec{p}_{2}\right) e^{\mathrm{i} k x \cdot \vec{n}_{2}} d s \\
& +\frac{\mathrm{i}}{\left(\vec{p}_{1} \cdot \vec{p}_{2}\right) k} \int_{B} q(x) \vec{p}_{2} \cdot E e^{\mathrm{i} k x \cdot \vec{n}_{2}} d x .
\end{aligned}
$$

From Theorem 2.1 and Remark 2.1, for a small wavenumber, the scattered field is weak and the inverse scattering problem becomes essentially linear. Dropping the nonlinear (second) term of (3.2), we obtain the linearized integral equation

$$
\int_{B} q_{0}(x) e^{\mathrm{i} k x \cdot\left(\vec{n}_{1}+\vec{n}_{2}\right)} d x=\frac{1}{\left(\vec{p}_{1} \cdot \vec{p}_{2}\right) k^{2}} \int_{S}(\nu \times E) \cdot\left(\vec{n}_{2} \times \vec{p}_{2}+\nu \times \vec{p}_{2}\right) e^{\mathrm{i} k x \cdot \vec{n}_{2}} d s,
$$

which is the Born approximation. The function $q_{0}(x)$ will be used as the starting point for our recursive linearization algorithm.

Since the scatterer $q_{0}(x)$ has a compact support, we use the notation

$$
\hat{q}_{0}(\xi)=\int_{B} q_{0}(x) e^{\mathrm{i} k x \cdot\left(\vec{n}_{1}+\vec{n}_{2}\right)} d x,
$$

where $\hat{q}_{0}(\xi)$ is the Fourier transform of $q_{0}(x)$ with $\xi=k\left(\vec{n}_{1}+\vec{n}_{2}\right)$. Choose

$$
\vec{n}_{j}=\left(\sin \theta_{j} \cos \phi_{j}, \sin \theta_{j} \sin \phi_{j}, \cos \theta_{j}\right), \quad j=1,2,
$$


where $\theta_{j}, \phi_{j}$ are the latitudinal and longitudinal angles, respectively. It is obvious that the domain $[0, \pi] \times[0,2 \pi]$ of $\left(\theta_{j}, \phi_{j}\right), j=1,2$, corresponds to the ball $\left\{\xi \in \mathbb{R}^{3}:|\xi| \leq\right.$ $2 k\}$. Thus, the Fourier modes of $\hat{q}_{0}(\xi)$ in the ball $\{\xi:|\xi| \leq 2 k\}$ can be determined. The scattering data with the higher wavenumber must be used in order to recover more modes of the true scatterer.

Define the data

$$
G(\zeta)= \begin{cases}\frac{1}{\left(\vec{p}_{1} \cdot \vec{p}_{2}\right) k^{2}} \int_{S}(\nu \times E) \cdot\left(\vec{n}_{2} \times \vec{p}_{2}+\nu \times \vec{p}_{2}\right) e^{\mathrm{i} k x \cdot \vec{n}_{2}} d s & \text { for }|\zeta| \leq 2 k, \\ 0, & \text { otherwise }\end{cases}
$$

where $\zeta=\zeta\left(k, \theta_{1}, \phi_{1}, \theta_{2}, \phi_{2}\right) \in \mathbb{R}^{3}$. The linear integral equation (3.3) can then be formally reformulated as

$$
\int_{\mathbb{R}^{3}} q_{0}(x) e^{\mathrm{i} x \cdot \zeta} d x=G(\zeta)
$$

Taking the inverse Fourier transform of (3.4) leads to

$$
\frac{1}{(2 \pi)^{3}} \int_{\mathbb{R}^{3}} e^{-\mathrm{i} x \cdot \zeta}\left[\int_{\mathbb{R}^{3}} q_{0}(y) e^{\mathrm{i} y \cdot \zeta} d y\right] d \zeta=\frac{1}{(2 \pi)^{3}} \int_{\mathbb{R}^{3}} e^{-\mathrm{i} x \cdot \zeta} G(\zeta) d \zeta .
$$

By the Fubini theorem, we have

$$
\frac{1}{(2 \pi)^{3}} \int_{\mathbb{R}^{3}} q_{0}(y)\left[\int_{\mathbb{R}^{3}} e^{\mathrm{i}(y-x) \cdot \zeta} d \zeta\right] d y=\frac{1}{(2 \pi)^{3}} \int_{\mathbb{R}^{3}} e^{-\mathrm{i} x \cdot \zeta} G(\zeta) d \zeta .
$$

Using the inverse Fourier transform of the Dirac delta function

$$
\frac{1}{(2 \pi)^{3}} \int_{\mathbb{R}^{3}} e^{\mathrm{i}(y-x) \cdot \zeta} d \zeta=\delta(y-x)
$$

we deduce

$$
\int_{\mathbb{R}^{3}} q_{0}(y) \delta(y-x) d y=\frac{1}{(2 \pi)^{3}} \int_{\mathbb{R}^{3}} e^{-\mathrm{i} x \cdot \xi} G(\xi) d \xi
$$

which gives

$$
q_{0}(x)=\frac{1}{(2 \pi)^{3}} \int_{\mathbb{R}^{3}} e^{-\mathrm{i} x \cdot \zeta} G(\zeta) d \zeta
$$

In practice, the integral equation (3.5) is implemented by using the FFT.

3.2. Recursive linearization. As discussed in the previous section, when the wavenumber is small, the Born approximation allows a reconstruction of those Fourier modes less than or equal to $2 k$ for the function $q(x)$. We now describe a procedure that recursively determines $q_{k}$ at $k=k_{j}$ for $j=1,2, \ldots$ with increasing wavenumbers. Suppose now that the scatterer $q_{\tilde{k}}$ has been recovered at some wavenumber $\tilde{k}$, and that the wavenumber $k$ is slightly larger than $\tilde{k}$. We wish to determine $q_{k}$, or equivalently, to determine the perturbation

$$
\delta q=q_{k}-q_{\tilde{k}}
$$


For the reconstructed scatterer $q_{\tilde{k}}$, we solve at the wavenumber $k$ the forward scattering problem

$$
\begin{aligned}
& \nabla \times(\nabla \times \tilde{E})-k^{2}\left(1+q_{\tilde{k}}\right) \tilde{E}=k^{2} q_{\tilde{k}} E^{i}, \quad x \in B, \\
& \nu \times(\nabla \times \tilde{E})+\mathrm{i} k \nu \times(\nu \times \tilde{E})=0 \quad \text { on } S .
\end{aligned}
$$

For the scatterer $q_{k}$, we have

$$
\begin{aligned}
& \nabla \times(\nabla \times E)-k^{2}\left(1+q_{k}\right) E=k^{2} q_{k} E^{i}, \quad x \in B, \\
& \nu \times(\nabla \times E)+\mathrm{i} k \nu \times(\nu \times E)=0 \quad \text { on } S .
\end{aligned}
$$

Subtracting (3.6), (3.7) from (3.8), (3.9) and omitting the second order smallness in $\delta q$ and in $\delta E=E-\tilde{E}$, we obtain

$$
\begin{aligned}
& \nabla \times(\nabla \times \delta E)-k^{2}\left(1+q_{\tilde{k}}\right) \delta E=k^{2} \delta q\left(E^{i}+\tilde{E}\right), \quad x \in B, \\
& \nu \times(\nabla \times \delta E)+\mathrm{i} k \nu \times(\nu \times \delta E)=0 \quad \text { on } S .
\end{aligned}
$$

For the scatterer $q_{k}$ and the incident wave $E^{i}$, we define the map $S\left(q_{k}, E^{i}\right)$ by

$$
S\left(q_{k}, E^{i}\right)=E,
$$

where $E$ is the scattered field at the wavenumber $k$. Let $\gamma$ be the trace operator to the boundary $S$ of the ball $B$. Define the scattering map

$$
M\left(q_{k}, E^{i}\right)=\gamma S\left(q_{k}, E^{i}\right) .
$$

It is easily seen that the scattering map $M\left(q_{k}, E^{i}\right)$ is linear with respect to $E^{i}$ but is nonlinear with respect to $q_{k}$. For simplicity, denote $M\left(q_{k}, E^{i}\right)$ by $M\left(q_{k}\right)$. By the definition of the trace operator, we have

$$
M\left(q_{k}\right)=\nu \times\left. E\right|_{S} .
$$

We refer to [1] for the Fréchet differentiability of the scattering map. Let $D M\left(q_{\tilde{k}}\right)$ be the Fréchet derivative of $M\left(q_{k}\right)$, and denote the residual operator

$$
R\left(q_{\tilde{k}}\right)=\nu \times\left.\delta E\right|_{S}
$$

It follows from [1] that

$$
D M\left(q_{\tilde{k}}\right) \delta q=R\left(q_{\tilde{k}}\right) .
$$

The regularized least-squares solution of (3.12) is

$$
\delta q=\left[\alpha I+D M^{*}\left(q_{\tilde{k}}\right) D M\left(q_{\tilde{k}}\right)\right]^{-1} D M^{*}\left(q_{\tilde{k}}\right) R\left(q_{\tilde{k}}\right),
$$

where $D M^{*}\left(q_{\tilde{k}}\right)$ is the adjoint operator of $D M\left(q_{\tilde{k}}\right), I$ is the identity operator, and $\alpha$ is some suitable positive number. In practice, the main difficulty is the enormous computational cost of solving linear systems with huge full matrix. Here, we consider an alternative way of solving (3.12) which is much less computationally demanding.

To state the approach, we first examine the boundary data $\nu \times E(x ; \theta, \phi ; k)$. Here, the variable $x$ is the observation point, which has two degrees of freedom since it is on the sphere $S$. The terms $\theta, \phi$ are latitudinal and longitudinal angles, respectively, of the incident wave $E^{i}$. At each frequency, we have four degrees of freedom, and thus 
data redundancy, which may be addressed by fixing one of the incident angles, say $\theta$. Define $\phi_{j}=(j-1) * \frac{2 \pi}{m}, j=1, \ldots, m$, and the residual operator

$$
R_{j}\left(q_{\tilde{k}}\right)=\nu \times\left. E\left(x ; \theta, \phi_{j} ; k\right)\right|_{S}-\nu \times\left.\tilde{E}\left(x ; \theta, \phi_{j} ; k\right)\right|_{S},
$$

where $m$ is the total number of the incident waves or sweeps, and $\tilde{E}\left(x ; \theta, \phi_{j} ; k\right)$ is the solution of (3.6), (3.7) with the incident wave of longitudinal angle $\phi_{j}$ and the scatterer $q_{\tilde{k}}$. Instead of solving (3.12) for all incident waves simultaneously, we may solve it for one incident wave at a time while updating the residual operator after each determination of the incremental correction $\delta q$. Thus, for each incident wave with incident angle $\phi_{j}$, we consider the equation

$$
M_{j}\left(q_{k}\right)=\nu \times\left. E\left(x ; \theta, \phi_{j} ; k\right)\right|_{S},
$$

where $M_{j}\left(q_{k}\right)$ is the scattering map corresponding to the incident wave with longitudinal angle $\phi_{j}$. It follows from [1] that

$$
D M_{j}\left(q_{\tilde{k}}\right) \delta q_{j}=R_{j}\left(q_{\tilde{k}}\right),
$$

where $D M_{j}\left(q_{\tilde{k}}\right)$ is the Fréchet derivative of the scattering map $M_{j}\left(q_{k}\right)$. The nonlinear Landweber iteration for (3.13) yields

$$
\delta q_{j}=\beta_{k} D M_{j}^{*}\left(q_{\tilde{k}}\right) R_{j}\left(q_{\tilde{k}}\right),
$$

where $D M_{j}^{*}\left(q_{\tilde{k}}\right)$ is the adjoint operator of $D M_{j}\left(q_{\tilde{k}}\right)$, and $\beta_{k}$ is some relaxation parameter [7].

Remark 3.1. For a fixed wavenumber, the stopping index of nonlinear Landweber iteration (3.15) could be determined from the discrepancy principle. However, in practice, it is not necessary to do many iterations. Our numerical results indicate that the iterative process for different incident angles $\phi_{j}, j=1, \ldots, m$, is sufficient to obtain reasonable accuracy.

Next, we discuss the role of the relaxation parameter $\beta_{k}$ in the iteration (3.15), which may be understood more clearly by considering the iteration from a different point of view.

Consider the optimization problem of (3.13),

$$
\min _{q_{k}}\left\|M_{j}\left(q_{k}\right)-\nu \times E\left(x ; \theta, \phi_{j} ; k\right)\right\|_{\left(L^{2}(S)\right)^{3}}^{2}
$$

The first order optimality condition for the problem (3.16) is given by

$$
\left.D M_{j}^{*}\left(q_{\tilde{k}}\right)\left(M_{j}\left(q_{k}\right)-\nu \times E\left(x ; \theta, \phi_{j} ; k\right)\right)\right|_{S}=0 .
$$

To solve the optimality equation (3.17), the time marching scheme proposed in [22] consists of finding the steady state of the following parabolic equation:

$$
\frac{d q_{k}}{d t}=\left.D M_{j}^{*}\left(q_{\tilde{k}}\right)\left(\nu \times E\left(x ; \theta, \phi_{j} ; k\right)-M_{j}\left(q_{k}\right)\right)\right|_{S} .
$$

The numerical solution could be computed from the explicit method

$$
\delta q_{j}=\tau D M_{j}^{*}\left(q_{\tilde{k}}\right) R_{j}\left(q_{\tilde{k}}\right)
$$


where $\tau$ is the discretized time step. Thus, the relaxation parameter $\beta_{k}$ is essentially the step size of time marching, whose length is restricted by the stability of the explicit method.

In order to compute the correction $\delta q_{j}$, we need some efficient way to compute $D M_{j}^{*}\left(q_{\tilde{k}}\right) R_{j}\left(q_{\tilde{k}}\right)$, which is given by the following theorem.

THEOREM 3.1. Given the residual $R_{j}\left(q_{\tilde{k}}\right)$, there exits a function $F_{j}$ satisfying the adjoint equations

$$
\begin{array}{r}
\nabla \times\left(\nabla \times F_{j}\right)-k^{2}\left(1+\overline{q_{\tilde{k}}}\right) F_{j}=0, \quad x \in B, \\
\nabla \times F_{j}-\mathrm{i} k \nu \times F_{j}=R_{j}\left(q_{\tilde{k}}\right) \quad \text { on } S,
\end{array}
$$

such that the adjoint Fréchet derivative $D M_{j}^{*}\left(q_{\tilde{k}}\right)$ satisfies

$$
\left[D M_{j}^{*}\left(q_{\tilde{k}}\right) R_{j}\left(q_{\tilde{k}}\right)\right](x)=k^{2}\left(\overline{E_{j}^{i}}(x)+\overline{\tilde{E}_{j}}(x)\right) \cdot F_{j}(x),
$$

where $E_{j}^{i}$ is the incident wave with the longitudinal angle $\phi_{j}$ and $\tilde{E}_{j}$ is the solution of (3.6), (3.7) with the incident wave $E_{j}^{i}$.

Proof. Let $\tilde{E}_{j}$ be the solution of (3.6), (3.7) with the incident wave $E_{j}^{i}$. Consider the equations as follows,

$$
\begin{aligned}
& \nabla \times(\nabla \times \delta E)-k^{2}\left(1+q_{\tilde{k}}\right) \delta E=k^{2} \delta q\left(E_{j}^{i}+\tilde{E}_{j}\right), \quad x \in B, \\
& \nu \times(\nabla \times \delta E)+\mathrm{i} k \nu \times(\nu \times \delta E)=0 \quad \text { on } S,
\end{aligned}
$$

and the adjoint equations (3.18) and (3.19), which take the variational form

$$
\begin{aligned}
\left(\nabla \times F_{j}, \nabla \times \phi\right)-k^{2}\left(\left(1+\overline{q_{\tilde{k}}}\right) F_{j}, \phi\right) & -\mathrm{i} k\left\langle\nu \times F_{j}, \nu \times \phi\right\rangle \\
& =\left\langle R_{j}\left(q_{\tilde{k}}\right), \nu \times \phi\right) \quad \forall \phi \in H_{\mathrm{imp}}(\operatorname{curl}, B) .
\end{aligned}
$$

The existence and uniqueness of the weak solution for the adjoint equations may be proved in the same way as for the scattered field. The proof is omitted.

Multiplying (3.21) with the complex conjugate of $F_{j}$ and integrating over $B$ on both sides, we obtain

$$
\int_{B} \bar{F}_{j} \cdot[\nabla \times(\nabla \times \delta E)] d x-k^{2} \int_{B}\left(1+q_{\tilde{k}}\right) \bar{F}_{j} \cdot \delta E d x=k^{2} \int_{B} \delta q\left(E_{j}^{i}+\tilde{E}_{j}\right) \cdot \bar{F}_{j} d x .
$$

Integration by parts yields

$$
\int_{S}\left[\delta E \times\left(\overline{\nabla \times F_{j}}\right)-\bar{F}_{j} \times(\nabla \times \delta E)\right] \cdot \nu d s=k^{2} \int_{B} \delta q\left(E_{j}^{i}+\tilde{E}_{j}\right) \cdot \bar{F}_{j} d x .
$$

Using the boundary condition (3.22), we deduce

$$
\int_{S}(\nu \times \delta E) \cdot\left(\overline{\nabla \times F_{j}+\mathrm{i} k \nu \times F_{j}}\right) d s=k^{2} \int_{B} \delta q\left(E_{j}^{i}+\tilde{E}\right) \cdot \bar{F}_{j} d x .
$$

It follows from (3.14) and the boundary condition (3.19) that

$$
\int_{S}\left[D M_{j}\left(q_{\tilde{k}}\right) \delta q\right] \cdot \overline{R_{j}\left(q_{\tilde{k}}\right)} d s=k^{2} \int_{B} \delta q\left(E_{j}^{i}+\tilde{E}_{j}\right) \cdot \bar{F}_{j} d x .
$$

We know from the adjoint operator $D M_{j}^{*}\left(q_{\tilde{k}}\right)$ that

$$
\int_{B} \delta q \overline{D M_{j}^{*}\left(q_{\tilde{k}}\right) R_{j}\left(q_{\tilde{k}}\right)} d x=k^{2} \int_{B} \delta q\left(E_{j}^{i}+\tilde{E}_{j}\right) \cdot \bar{F}_{j} d x
$$


TABLE 1

Recursive linearization reconstruction algorithm for inverse medium scattering.

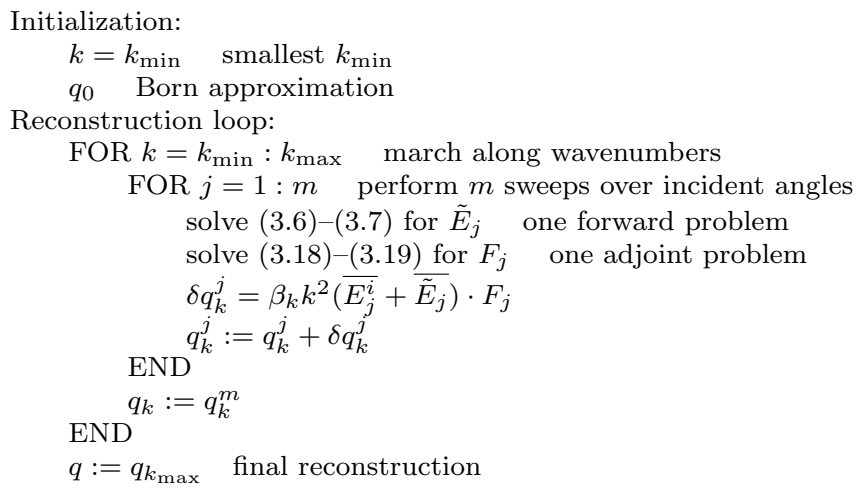

Since this holds for any $\delta q$, we have

$$
\overline{D M_{j}^{*}\left(q_{\tilde{k}}\right) R_{j}\left(q_{\tilde{k}}\right)}=k^{2}\left(E_{j}^{i}+\tilde{E}_{j}\right) \cdot \bar{F}_{j} .
$$

Taking the complex conjugate of the above equation yields the result.

Using this theorem, we can rewrite (3.15) as

$$
\delta q_{j}=\beta_{k} k^{2}\left(\overline{E_{j}^{i}}(x)+\overline{E_{j}}(x)\right) \cdot F_{j}(x) .
$$

Thus, for each incident wave with a longitudinal angle $\phi_{j}$, we solve one forward problem (3.6), (3.7) and one adjoint problem (3.18), (3.19). Since the adjoint problem has a variational form similar to that of the forward problem, we need to compute essentially two forward problems at each sweep. Once $\delta q_{j}$ is determined, $q_{\tilde{k}}$ is updated by $q_{\tilde{k}}+\delta q_{j}$. After completing the $m$ th sweep, we get the reconstructed scatterer $q_{k}$ at the wavenumber $k$.

The recursive linearization for inverse medium scattering of Maxwell's equations can be summarized in Table 1 .

4. Numerical experiments. In this section, we discuss the numerical solution of the forward scattering problem and the computational issues of the recursive linearization algorithm.

As for the forward solver, we adopt the edge elements which were developed originally for the finite element solution of Maxwell's equations [20, 12] in the early 1980s. From the mathematical point of view, these are natural approximation spaces for the Hilbert space $H(\operatorname{curl}, B)$, which is the adequate functional space for the variational formulation of Maxwell's equations. Vector fields in such finite element (FE) spaces have continuous tangential traces, which is consistent with the physics. Therefore, the natural degrees of freedom for these elements are related to tangential traces along edges or faces. Here, we take the symmetric second order edge elements for tetrahedral edge elements [13]. When the unknowns are ordered according to the reverse Cuthill-McKee (RCM) ordering [9], the profile of FE matrix is highly banded, which improves the condition number of the FE coefficient matrix. The sparse large scale linear system can be most efficiently solved if the zero elements of the coefficient matrix are not stored. We use the commonly used compressed row storage (CRS) 


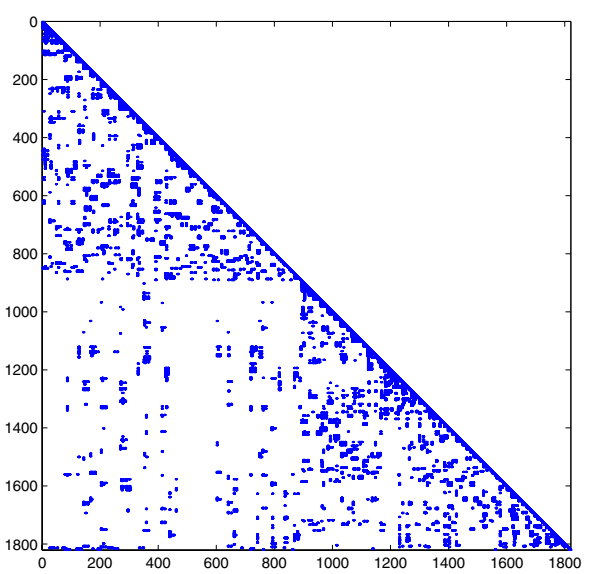

(a)

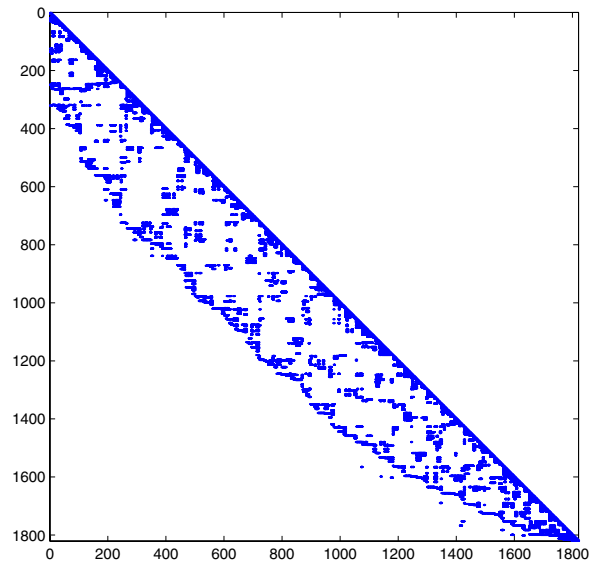

(b)

FIG. 1. Sparsity pattern of an FE matrix with 1820 unknowns: (a) original ordering, (b) RCM ordering.

format, which makes no assumptions about the sparsity structure of the matrix and does not store any unnecessary elements. In fact, from the variational formula of our direct problem (2.1), the coefficient matrix is complex symmetric. Hence, only the lower triangular portion of the matrix needs to be stored. Figure 1 shows a typical sparsity pattern of an FE matrix with 1820 unknowns from the symmetric second order edge element. Regarding the linear solver, either biconjugate gradient (BiCG) or quasi-minimal residual (QMR) algorithms with diagonal preconditioning may be employed to solve the sparse, symmetric, and complex system of the equations. It appears for our experiments that the QMR is more efficient.

In the following, we present two numerical examples where the number of the incident wave $m=20$, the incident latitudinal angle $\theta=0$, and the incident longitudinal angle $\phi_{j}=(j-1) * \frac{2 \pi}{m}, j=1, \ldots, m$. The relaxation parameter $\beta_{k}$ is taken to be $0.1 / k$ for the tested examples. For stability analysis, some relative random noise is added to the data; i.e., the tangential trace of the electric field takes the form

$$
\nu \times\left. E\right|_{S}:=(1+\sigma \text { rand }) \cdot\left(\nu \times\left. E\right|_{S}\right) .
$$

Here, rand gives uniformly distributed random numbers in $[-1,1]$, and $\sigma$ is a noise level parameter taken to be 0.02 in our numerical experiments. Define the relative error by

$$
e_{2}=\frac{\left(\sum_{i, j, k}\left|q_{i j k}-\bar{q}_{i j k}\right|^{2}\right)^{\frac{1}{2}}}{\left(\sum_{i, j, k}\left|q_{i j k}\right|^{2}\right)^{\frac{1}{2}}},
$$

where $\bar{q}$ is the reconstructed scatter and $q$ is the true scatterer.

Example 4.1. Reconstruct a scatterer defined by

$$
q(x, y, z)= \begin{cases}1-\sqrt{\frac{x^{2}}{1^{2}}+\frac{y^{2}}{0.8^{2}}+\frac{z^{2}}{0.5^{2}}} & \text { for } \frac{x^{2}}{1^{2}}+\frac{y^{2}}{0.8^{2}}+\frac{z^{2}}{0.5^{2}} \leq 1, \\ 0, & \text { otherwise. }\end{cases}
$$

The compact support of this scatterer is an ellipsoid contained in the unit ball. For simplicity, we take $\vec{n}_{1}=\vec{n}_{2}$ and $\vec{p}_{1}=\vec{p}_{2}$ to test the forward solver. The numerical 


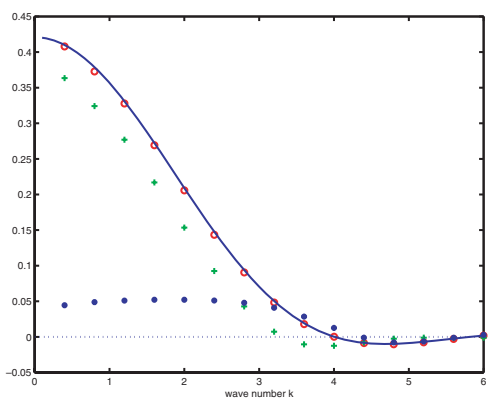

(a)

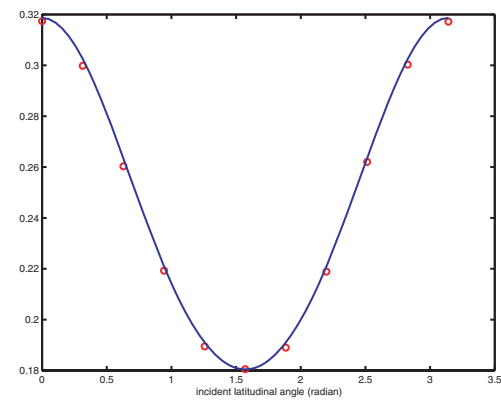

(b)

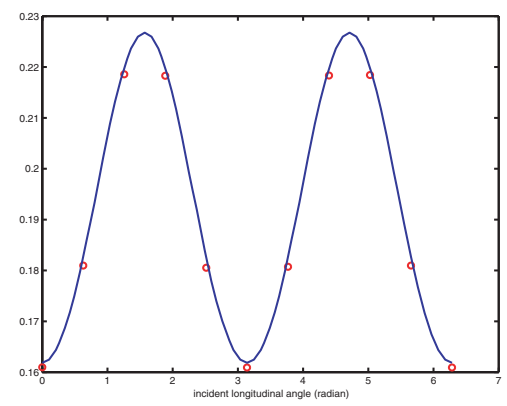

(c)

FIG. 2. (a) Integrals at different wavenumbers for the fixed incident angle $\theta=\frac{\pi}{3}$ and $\phi=\frac{\pi}{3}$. Solid curve: the exact integral value of the left-hand side of (3.2), +: the computed integral value of the first term of the right-hand side of (3.2), *: the computed integral value of the second term of right-hand side of (3.2), ०: the computed integral value of the right-hand side of (3.2). (b) Integrals with different $\theta$ for the fixed wavenumber $k=2.0$ and $\phi=\frac{\pi}{3}$. Solid curve: the exact integral value of the left-hand side of (3.2), o: the computed integral value of the right hand-side of (3.2). (c) Integrals with different $\phi$ for the fixed wavenumber $k=2.0$ and $\theta=\frac{\pi}{3}$. Solid curve: the exact integral value of the left-hand side of (3.2), ०: the computed integral value of the right-hand side of (3.2).

TABLE 2

Relative error at different wavenumbers.

\begin{tabular}{ccccccc}
\hline$k$ & 1 & 2 & 3 & 4 & 5 & 6 \\
\hline$e_{2}$ & 0.5494 & 0.4876 & 0.3197 & 0.1856 & 0.1534 & 0.0895 \\
\hline
\end{tabular}

results are shown in Figure 2. In Figure 2(a), for the fixed incident latitudinal angle $\theta=\frac{\pi}{3}$ and the longitudinal angle $\phi=\frac{\pi}{3}$, the forward problem is solved at different wavenumbers. In Figure 2(b) and 2(c), for the fixed wavenumber $k=2$, the numerical results are shown with different latitudinal angles $\theta \in[0, \pi]$ (fix $\phi=\frac{\pi}{3}$ ) and $\phi \in[0,2 \pi]$ (fix $\theta=\frac{\pi}{3}$ ), respectively. It is easily seen from Figure 2(a) that the first term of the right-hand side of the integral equation (3.2) is dominant compared with the second (nonlinear) term when the wavenumber is small, which validates the Born approximation. Figure 3 shows the slices of the true scatterer, and Figure 4 gives the reconstruction at the wavenumber $k=6$. The relative errors are shown in Table 2 at different wavenumbers. 


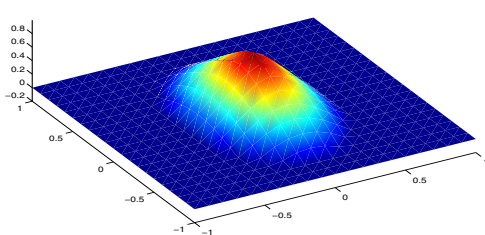

(a)

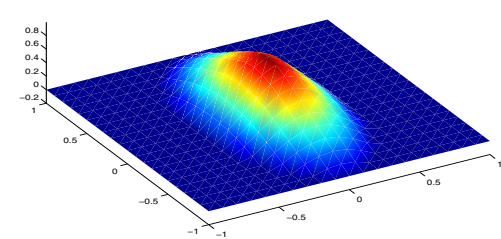

(b)

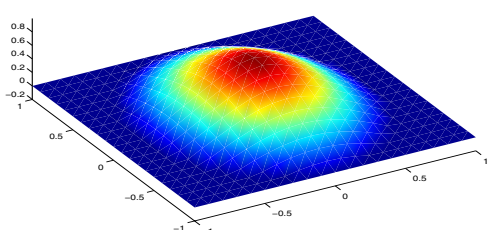

(c)

FIG. 3. True scatterer of Example 4.1: (a) the slice $x=0$; (b) the slice $y=0$; (c) the slice $z=0$.

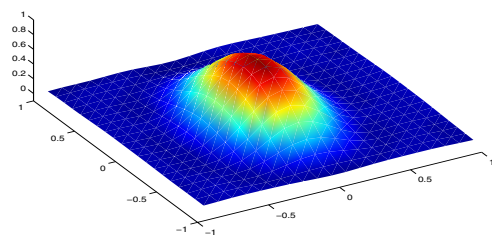

(a)

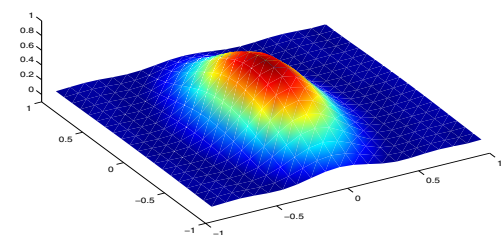

(b)

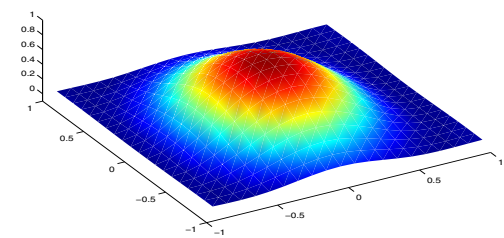

(c)

FiG. 4. Reconstruction of Example 4.1: (a) the slice $x=0$; (b) the slice $y=0$; (c) the slice $z=0$.

Example 4.2. Reconstruct a scatterer defined by

$q(x, y, z)= \begin{cases}\sin \left(\frac{4 \pi}{25}\right)-\sin \left(\left(x^{2}+(y+0.5)^{2}+z^{2}\right) \pi\right) & \text { for } x^{2}+(y+0.5)^{2}+z^{2} \leq 0.4^{2}, \\ \sin \left(\frac{4 \pi}{25}\right)-\sin \left(\left(x^{2}+(y-0.5)^{2}+z^{2}\right) \pi\right) & \text { for } x^{2}+(y-0.5)^{2}+z^{2} \leq 0.4^{2} \\ 0, & \text { otherwise }\end{cases}$

The compact support of this scatterer is two isolated balls with the same radius of 0.4 and the centers at $(0,-0.5,0)$ and $(0,0.5,0)$. For simplicity, we take $\vec{n}_{1}=\vec{n}_{2}$ 


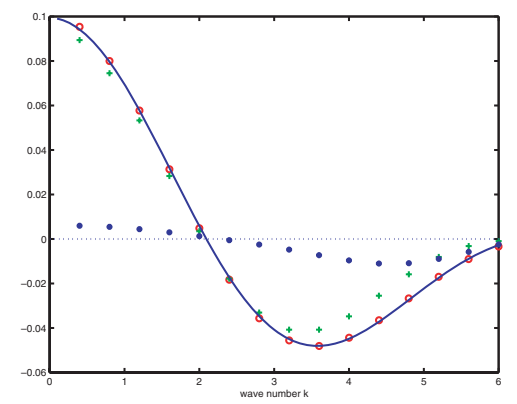

(a)

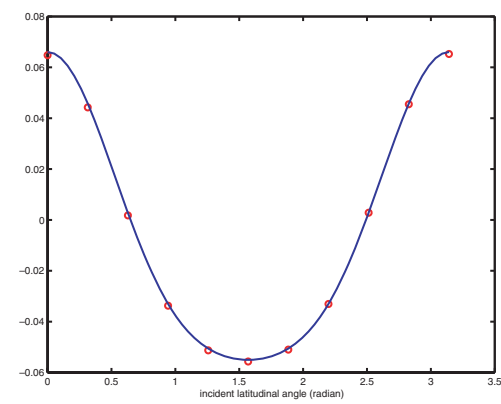

(b)

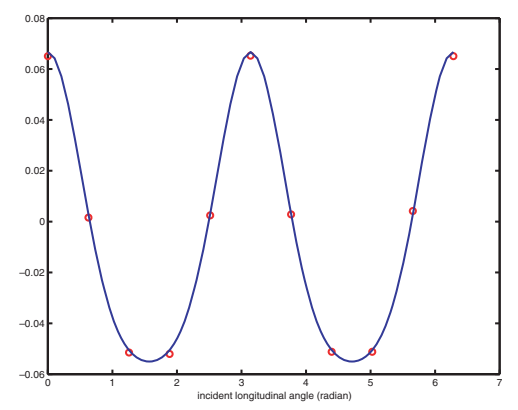

(c)

FIG. 5. (a) Integrals with different wavenumbers for the fixed incident angle $\theta=\frac{\pi}{3}$ and $\phi=\frac{\pi}{3}$. Solid curve: the exact integral value of the left-hand side of (3.2), +: the computed integral value of the first term of the right-hand side of (3.2), *: the computed integral value of the second term of right-hand side of (3.2), ०: the computed integral value of the right-hand side of (3.2). (b) Integrals with different $\theta$ for the fixed wavenumber $k=3.0$ and $\phi=\frac{\pi}{3}$. Solid curve: the exact integral value of the left-hand side of (3.2), o: the computed integral value of the right hand-side of (3.2). (c) Integrals with different $\phi$ for the fixed wavenumber $k=3.0$ and $\theta=\frac{\pi}{3}$. Solid curve: the exact integral value of the left-hand side of (3.2), o: the computed integral value of the right-hand side of (3.2).

TABLE 3

Relative error at different wavenumbers.

\begin{tabular}{cccccccc}
\hline$k$ & 1 & 2 & 3 & 4 & 5 & 6 & 7 \\
\hline$e_{2}$ & 0.6963 & 0.6479 & 0.5891 & 0.4951 & 0.3376 & 0.2568 & 0.2221 \\
\hline
\end{tabular}

and $\vec{p}_{1}=\vec{p}_{2}$ in the test of the forward solver. The numerical results are given in Figure 5. In Figure 5(a), for the fixed incident latitudinal angle $\theta=\frac{\pi}{3}$ and the longitudinal angle $\phi=\frac{\pi}{3}$, the forward problem is solved at different wavenumbers. In Figure 5(b) and 5(c), for the fixed wavenumber $k=3$, the numerical results are shown with different latitudinal angles $\theta \in[0, \pi]$ (fix $\phi=\frac{\pi}{3}$ ) and $\phi \in[0,2 \pi]$ (fix $\left.\theta=\frac{\pi}{3}\right)$, respectively. It is easily seen from Figure 5(a) that the first term of the right-hand side of the integral equation (3.2) is dominant compared with the second (nonlinear) term when the wavenumber $k$ is small, which once again validates the Born approximation. Figure 6 shows the slices of the true scatterer and Figure 7 gives the reconstruction at the wavenumber $k=7$. The relative errors are shown in Table 3 at different wavenumbers.

5. Concluding remarks. The proposed recursive linearization algorithm is stable and efficient for solving the inverse medium scattering problem with multiple 


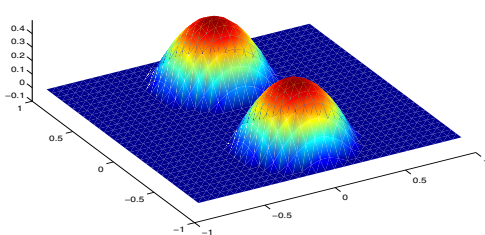

(a)

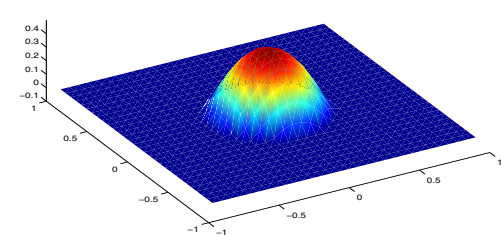

(b)

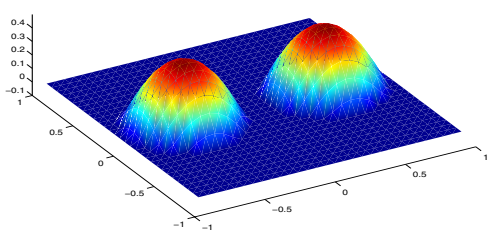

(c)

FIG. 6. True scatterer of Example 4.2: (a) the slice $x=0$; (b) the slice $y=-0.5$; (c) the slice $z=0$.

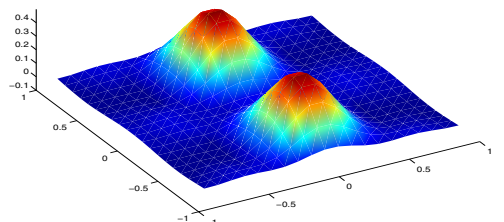

(a)

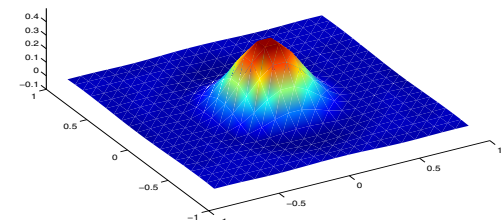

(b)

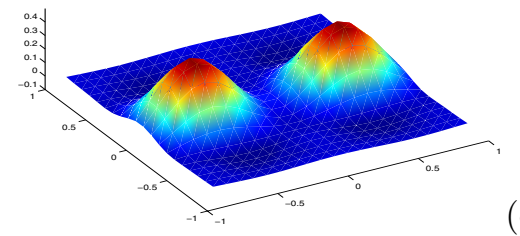

FIG. 7. Reconstruction of Example 4.2: (a) the slice $x=0$; (b) the slice $y=-0.5$; (c) the slice $z=0$.

frequency scattering data in three dimensions. Theoretically, scattering data with even higher wavenumbers could be used to recover more complicated scatterers which contain higher-frequency features, i.e., more Fourier modes. However, the difficulty lies in the fact that the forward model becomes difficult to solve due to the highly oscillatory nature of the solution. For a larger $k$, the mesh size has to be smaller, which makes numerical solution more expensive. Finally, we point out two important future directions of this research. The first concerns the convergence analysis of the 
recursive linearization algorithm, which is currently in progress and will be reported elsewhere. Another challenging project is to develop an efficient algorithm for the inverse medium scattering with fixed frequency scattering data.

\section{REFERENCES}

[1] H. Ammari And G. Bao, Analysis of the scattering map of a linearized inverse medium problem for electromagnetic waves, Inverse Problems, 17 (2001), pp. 219-234.

[2] G. BAO AND J. LiU, Numerical solution of inverse scattering problems with multi-experimental limited aperture data, SIAM J. Sci. Comput., 25 (2003), pp. 1102-1117.

[3] G. BAO AND P. LI, Inverse medium scattering for three-dimensional time harmonic Maxwell equations, Inverse Problems, 20 (2004), pp. L1-L7.

[4] Y. CHEN, Inverse scattering via Heisenberg uncertainty principle, Inverse Problems, 13 (1997), pp. 253-282.

[5] D. Colton And R. KRess, Inverse Acoustic and Electromagnetic Scattering Theory, 2nd ed., Appl. Math. Sci. 93, Springer-Verlag, Berlin, 1998.

[6] O. Dorn, H. Bertete-Aguirre, J. G. Berryman, and G. C. Papanicolaou, A nonlinear inversion method for $3 D$ electromagnetic imaging using adjoint fields, Inverse Problems, 15 (1999), pp. 1523-1558.

[7] H. Engl, M. Hanke, and A. Neubauer, Regularization of Inverse Problems, Kluwer Academic Publisher, Dordrecht, The Netherlands, 1996.

[8] M. Eller, V. Isakov, G. Nakamura, And D. Tataru, Uniqueness and stability in the Cauchy problem for Maxwell's and elasticity system, in Proceedings of Nonlinear Partial Differential Equations and Their Applications 14 (College de France Seminar), D. Cioranescu and J. L. Lions, eds., Stud. Math. Appl., 31, North-Holland, Amsterdam, 2002, pp. 329-350.

[9] N. E. Gibbs, W. G. Poole, JR., And P. K. Stockmeyer, An algorithm for reducing the bandwidth and profile of a sparse matrix, SIAM J. Numer. Anal., 13 (1976), pp. 236-250.

[10] T. HoHAGE, On the numerical solution of a three-dimensional inverse medium scattering problem, Inverse Problems, 17 (2001), pp. 1743-1763.

[11] H. HADDAR AND P. MONK, The linear sampling method for solving the electromagnetic inverse medium problem, Inverse Problems, 18 (2002), pp. 891-906.

[12] J. Jin, The Finite Element Methods in Electromagnetics, John Wiley \& Sons, New York, 2002.

[13] A. KAMEARI, Symmetric second order edge elements for triangle and tetrahedra, IEEE Trans. Magn., 35 (1999) pp. 1394-1397.

[14] A. Kirsch AND P. Monk, A finite element/spectral method for approximating the timeharmonic Maxwell system in $\mathbb{R}^{3}$, SIAM J. Appl. Math., 55 (1995), pp. 1324-1344.

[15] A. Kirsch And P. Monk, Corrigendum: A finite element/spectral method for approximating the time-harmonic Maxwell system in $\mathbb{R}^{3}$, SIAM J. Appl. Math., 58 (1998), pp. 2024-2028.

[16] A. KIRsch And P. Monk, A finite element method for approximating electro-magnetic scattering from a conducting object, Numer. Math., 92 (2002), pp. 501-534.

[17] P. Monk, Finite Element Methods for Maxwell's Equations, Oxford University Press, Oxford, U.K., 2003.

[18] F. NATTERER, The Mathematics of Computerized Tomography, Teubner, Stuttgart, 1986.

[19] F. Natterer and F. WüBbeling, A propagation-backpropagation method for ultrasound tomography, Inverse Problems, 11 (1995), pp. 1225-1232.

[20] J. C. NÉDÉLEC, Mixed finite elements in $\mathbb{R}^{3}$, Numer. Math., 35 (1980), pp. 315-341.

[21] J. C. NÉDÉLEC, Acoustic and Electromagnetic Equations: Integral Representations for Harmonic Problems, Springer, New York, 2000.

[22] L. Rudin, S. OsheR, AND E. FAtemi, Nonlinear total variation based noise removal algorithms, Phys. D, 60 (1992), pp. 259-268.

[23] M. VöGELER, Reconstruction of the three-dimensional refractive index in electromagnetic scattering by using a propagation-backpropagation method, Inverse Problems, 19 (2003), pp. 739-753. 